\title{
Applying blockchain technology to the Australian carbon market
}

\author{
Sam Hartmann ${ }^{1}$ and Sebastian Thomas ${ }^{1,2 *}$
}

${ }^{1}$ Sustainability Science Lab, University of Melbourne

${ }^{2}$ School of Ecosystem and Forest Sciences, University of Melbourne

* Corresponding author: sebastian.thomas@unimelb.edu.au

G58b, Baldwin Spencer West (113)

University of Melbourne

Parkville VIC 3010

AUSTRALIA

\begin{abstract}
Blockchain is a distributed digital ledger system that establishes transparent contract processes and facilitates secure but trusted business transactions. Policymakers around the world are intrigued by the potential of this emerging technology to solve policy problems, including the challenges of the transition away from centralised, linear models of energy generation and consumption toward decentralised and distributed energy systems. Blockchain has also been promoted as a mechanism to transform carbon markets, yet the focus in this area to date has been on using blockchain to create new carbon market schemes. This paper addresses an important research gap by asking how blockchain could be applied to an existing carbon market. To answer this question, the study uses an established design process to develop an Australian carbon market blockchain design. The paper finds that this design could improve the efficiency, equity, and effectiveness of the Australian carbon market. This paper makes an important research contribution to carbon market policy development by developing a blockchain design that could improve how an existing carbon market functions, and the findings presented here are relevant to government and industry stakeholders globally.
\end{abstract}

This is the author manuscript accepted for publication and has undergone full peer review but has not been through the copyediting, typesetting, pagination and proofreading process, which may lead to differences between this version and the Version of Record. Please cite this article as doi: $10.1111 / 1759-3441.12266$

This article is protected by copyright. All rights reserved 


\section{Keywords}

Distributed ledger; decentralised energy; emissions trading; technological innovation.

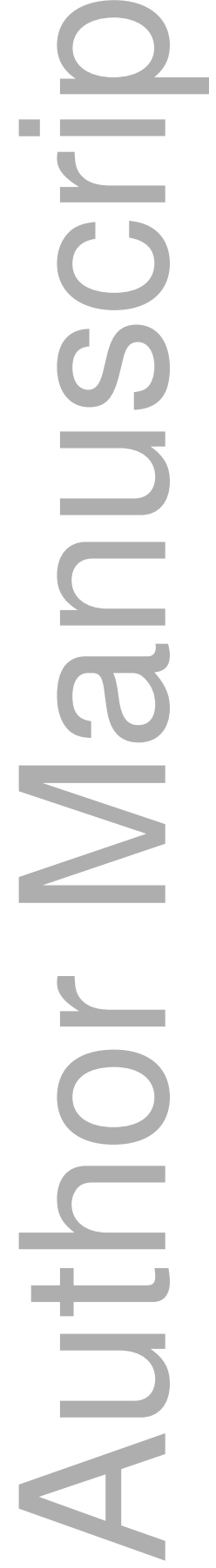


2 DR. SEBASTIAN THOMAS (Orcid ID : 0000-0002-3345-9120)

3

4

5

6

7

8

9

10

11

12

13

14

15

Article type : Special Section

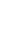

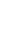

\section{Applying blockchain to the Australian carbon market} (n)

(1)

\section{Introduction}

2

Blockchain is an emerging technology that promises to transform how individuals, industry, and government operate (Green and Newman, 2017). Blockchain is a distributed ledger supported by a network that conducts, validates, and records its transactions. The first blockchain was introduced by Nakamoto (2008) in the Bitcoin electronic payment system. Bitcoin was founded on the belief that the incumbent approach for untrusted parties to conduct transactions using a trusted authority was more hindrance than help (Nakamoto, 2008). Instead, Nakamoto (2008) proposed an open decentralised system backed by cryptography to embed trust in the network itself (Suhas et al., 2015; Xu et al., 2017). Bitcoin has demonstrated stability during periods of economic turbulence and is considered a potential hedge against financial instability (Bouri et al., 2017; Selmi et al., 2018). Policymakers around the world are therefore intrigued by blockchain technologies and are beginning to explore their potential to solve public policy problems (Alketbi et al., 2018). This exploration extends to climate action and electricity and carbon markets where there is enthusiasm for the role blockchain could play (United Nations Climate Change, 2017; Green and Newman, 2017; United Nations Climate Change, 2018; Dong et al., 2018; Diestelmeier, 2019)

The blockchain process begins with parties directly conducting peer to peer transactions with no intermediary. These transactions can transfer digital assets or information through simple protocols, or incorporate complex programming known as smart contracting. These smart contracts automatically execute and transfer digital assets according to predefined 
1 programmed rules (Buterin, 2013). Each party holds a copy of the blockchain ledger that they can use to verify the transaction, and once satisfied broadcast the transaction to the network. The network collects and verifies the broadcasted transactions into a chronological list known as a 'block'. The network then undertakes a consensus process to agree on adding the current block of transactions to the ledger. Upon the network reaching consensus, the block is connected or 'chained' to all proceeding blocks. The block is chained using a practically irreversible cryptographic process known as hashing. Hashing summarises the ledger data into a concise string of characters that warns if the underlying data is changed. Each copy of the distributed ledger is then updated and a new block created that incorporates the newly minted hash. The network then moves onto the next block and the process continues.

Blockchain's attraction lies in its data immutability, transparency, verification, and privacy and security (Aste et al., 2017, Catalini and Gans, 2017). The blockchain delivers a single practically tamper-proof transaction history that promotes network confidence and guards against transaction disavowal (Aste et al., 2017). This history comes with transparency that enables traceability (Kristoffer and David, 2018), and auditability that provides fraud and corruption protection (Kshetri, 2017). As a transaction system it can reduce costs in verification (Catalini and Gans, 2017) and in maintaining multiple ledgers (Aste et al., 2017; Iansiti and Lakhani, 2017). However, blockchain is accompanied by scepticism of whether it can live up to its promises (Narayanan and Clark, 2017). Blockchain's decentralised ethos is threatened by consolidation pressures (Aste et al., 2017). There are also cost and scalability concerns (Aste et al., 2017; Xu et al., 2017), and questions as to whether blockchain only redistributes rather than reduces privacy and security risks (Xu et al., 2017; Iansiti and Lakhani, 2017; Narayanan and Clark, 2017). Ultimately, however, blockchain's potential is driving the exploration of new use cases, including electricity and carbon markets (e.g. Diestelmeier, 2019).

While distributed energy systems and technologies to support new economic models of electricity generation and consumption are being investigated (William et al., 2012; Burger and Luke, 2017; Green and Newman, 2017), exactly how blockchain could be applied to carbon markets has only just begun to be explored. Carbon markets are premised on the theory that reducing emissions can be more economically and environmentally effectively achieved through trading (Bumpus and Liverman, 2008; Schmalensee and Stavins, 2017). Carbon markets include both emissions trading schemes (ETS) and offset schemes. ETS set 
1 an emissions limit, allocate emissions allowances and permit trading of allowances until they are used to meet compliance obligations (MacKenzie, 2009). Offset schemes issue carbon

3

4 credits for projects that reduce, sequester, or avoid emissions (MacKenzie, 2009). These projects report to an authority that issues carbon credits, that can then be retired or used to meet compliance obligations (MacKenzie, 2009). Supporting these carbon markets schemes are accounting systems, rules and institutions that may be suitable for blockchain technology (Dong et al., 2018). The enthusiasm for how blockchain might be applied to carbon markets is outpacing research. To date, the focus of published research has been on creating new carbon market schemes using blockchain. The application of blockchain to existing carbon market schemes remains unexplored in the literature. With 25 existing carbon markets worldwide (World Bank and Ecofys, 2018) it is important to understand how blockchain might be applied to these schemes.

This paper fills the research gap by asking how blockchain could be applied to an existing carbon market. The paper selects the Australian carbon market as its case study. Through a systematic literature review the paper establishes four proposed blockchain carbon market applications. To apply blockchain to an existing carbon market this paper adopts the blockchain system design process proposed by Xu et al. (2017). This paper then assesses the design features from the literature's four proposed models against the Australian carbon market. Through this process a recommended Australian carbon market blockchain design is developed. The paper concludes by finding that adopting this blockchain design could improve the functioning of the Australian carbon market.

\section{Literature Review}

As a recent phenomenon, it is unsurprising that there is only limited literature on applying blockchain to carbon markets. Through a systematic literature review it was found that blockchain carbon market applications were in four clusters. Under each of these four clusters the respective blockchain models will be introduced. These clusters are:

- a 'Networked Carbon' cluster (Macinante, 2016, Macinante, 2017, Jackson et al., 2017, Dong et al., 2018); 
- an 'Industry 4.0' cluster focusing on automation and digitisation (Fu et al., 2018, Khaqqi et al., 2018);

- a 'Technolibertarian' anonymous carbon trading cluster (Al Kawasmi et al., 2015); and

- an isolationist smart contract based 'Voluntary Offset' cluster (Leonhard, 2017a, Leonhard, 2017b).

The four clusters operate independently in the literature with focus on creating new carbon market schemes. This reveals a clear research gap in exploring how blockchain could be applied to existing carbon markets. This paper fills this gap by asking its key research question of how blockchain could be applied to an existing carbon market.

\subsection{Networked Carbon}

Networked carbon markets are based on connecting separate carbon markets to a larger global carbon market. This cluster's literature proposes that each jurisdiction would maintain its own carbon market that connects and trades with others through an overarching blockchain (Macinante, 2017, Jackson et al., 2017, Dong et al., 2018). This Networked Carbon model is classified as a partially decentralised, permissioned, smart contract enabled blockchain.

\subsection{Industry 4.0}

The Industry 4.0 cluster focuses on embracing blockchain's automation and digitisation. The Industry 4.0 cluster proposes using automation from smart devices to support monitoring, reporting and verification (MRV) in carbon markets. The cluster also uses the blockchain to incorporate into new carbon market features such as firm reputations and trading restrictions (Khaqqi et al., 2018), and sectoral supply chains (Fu et al., 2018). The Industry 4.0 model is classified as a partially decentralised permissioned blockchain model.

\subsection{Technolibertarian}

The Technolibertarian cluster is aimed at attracting otherwise reluctant participants, due to privacy and security concerns, into a voluntary carbon market (Al Kawasmi et al., 2015). This Technolibertarian model takes the Bitcoin model as its foundation. It supplements Bitcoin by introducing a primitive version of smart contracting through a platform that processes, validates and stores contracts. This model also proposes that through smart devices 
that a blockchain could produce its own carbon credits for trading. This Technolibertarian model is classified as an open, public blockchain with decentralised consensus (Al Kawasmi et al., 2015).

\subsection{Voluntary Offset}

The Voluntary Offset cluster proposes conducting offset projects using smart contracts. This cluster leverages off the Ethereum blockchain to create Decentralised Autonomous Organisations (DAOs) (Buterin, 2013). These DAOs house voluntary offset projects. The DAOs are managed by Universities and raise capital through Initial Coin Offerings (Aste et al., 2017; Leonhard, 2017b). The offset project is then implemented, verified by another University, before providing its carbon credits to its investors (Leonhard, 2017b). The second variant of this proposal groups multiple offsets projects under larger DAOs (Leonhard, 2017a). This Voluntary Offset model is classified as a partially decentralised smart contractbased blockchain model.

\section{Methods}

To answer how blockchain could be applied to an existing carbon market, this paper first introduces the Australian carbon market. To determine how blockchain could be applied to this market, this paper uses the blockchain design process proposed by $\mathrm{Xu}$ et al. (2017). The design process considers the key blockchain features and its accompanying design options. This design process enables a systematic consideration of blockchain design features and trade-offs (Xu et al., 2017). This paper uses document analysis as its principal investigative technique. The data to support this analysis is collected from the systematic literature review in Appendix A, and combined with peer reviewed and grey literature, and publicly available information on the Australian carbon market. The literature's four models are assessed against the existing Australian carbon market to develop the recommended Australian carbon market blockchain design. The existing Australian carbon market rules, legislation, institutions and infrastructure are all considered as fixed constraints in the development of this blockchain design.

\subsection{Design process for blockchain-based systems}

Xu et al.'s (2017) design process guides the application of blockchain to the Australian carbon market. The design process contains four major steps. The steps cover decentralisation decisions, storage and computation decisions, blockchain configuration 
1 decisions and other design decisions (Figure 1). Within each step there are discrete design 2 decisions (Table 1).

3

4 Figure 1 - Design process for blockchain-based systems (Xu et al. 2017)

5

6

7 The design process starts with the trust decentralisation decision. The initial consideration is 8 9 whether there is a trusted authority in the network. The presence of the trusted authority determines whether a decentralisation is required. If there is a trusted authority, decisions are made about the possibility of decentralisation or use of traditional databases. If trusted authority functions can be decentralised, then decisions will need to be made on how it could be enacted. This includes any permissions and verifier roles within the blockchain.

The second step concerns blockchain data storage and computation. The first design decision is around whether item data and item collections should be stored 'on-chain' (i.e. on the blockchain) or stored externally 'off-chain' (i.e. outside of the blockchain). The second design decision considers whether the computation should be simple transaction constraints or complex smart contracting. This decision also considers whether the computation should occur on chain or off chain.

The third step consists of blockchain configuration decisions. The first decision is around whether the blockchain should be public (i.e. open to all), consortium-based (i.e. open across multiple organisations) or private (i.e. restricted access). The second decision is whether the data structure should be a conventional blockchain data block structure or another structure. The third decision is the consensus protocol that reaches agreement to update the blockchain. This protocol can then be tailored to promote security or scalability in the fourth design decision. The fifth design decision concerns whether to create a new blockchain or connect it to existing blockchains. The sixth design decision is whether to use multiple blockchains.

The fourth step captures other design decisions. These decisions include the degree of anonymity within the network and whether the system contains incentives to support system operation. The last design decision is deciding how the blockchain could be deployed.

\section{Table 1 here - Blockchain design decisions (Xu et al., 2017)}




\section{The Australian carbon market}

\subsection{Policy context}

6 Australian climate change policy is focused on using market-based mechanisms to reduce 7 emissions at the lowest cost (Commonwealth of Australia, 2008, Commonwealth of 8 Australia, 2011, Commonwealth of Australia, 2014). The current incarnation of the 9 Australian carbon market is an economy-wide voluntary carbon offset scheme under the Emissions Reduction Fund (ERF) (Commonwealth of Australia, 2014). The ERF has three components; crediting, purchasing, and safeguarding emissions reductions (Commonwealth of Australia, 2014). To credit emissions reductions, the ERF used the infrastructure from the earlier Carbon Farming Initiative (CFI). This included retaining, but rebranding, the CFI's method development and approval processes, offset projects and methods, legal arrangements, Australian Carbon Credit Units (ACCUs), and Clean Energy Regulator (the Regulator) (Commonwealth of Australia, 2014). The ERF purchasing component consists of the Regulator contracting offset projects through a reverse auction process. The ERF purchasing component was $\$ 2.55$ billion of funding from 2014-15 to 2018-19 (Commonwealth of Australia, 2014). The Regulator selects offset projects based on price to enter into standardised contracts to buy ACCUs (Commonwealth of Australia, 2014). The safeguard mechanism provides a limit on emissions for the largest emitters based on historical baselines (Commonwealth of Australia, 2014). If liable firms exceed their baselines they can purchase ACCUs to reduce their net emissions or face compliance actions (Department of the Environment, 2016).

\subsection{Carbon market scope}

27 This paper defines the Australian carbon market as the trading of ACCUs. This Australian carbon market has developed into one of the world's largest offset trading schemes (Commonwealth of Australia, 2017b). It has its ERF primary market, where the Australian Government has paid or committed nearly $\$ 2.3$ billion to 461 offset projects to deliver 192 million ACCUs (Clean Energy Regulator, 2018a). The Australian carbon market also has a growing secondary market with an estimated 2 million ACCUs available for trading (Climate 
1 ERF contracts, firm liabilities from the safeguard mechanism, and limited voluntary market demand (Climate Change Authority, 2017).

3

\section{$4 \quad 4.3$ Carbon market performance}

5 The Australian carbon market contains a reasonably well performing ERF but a languishing 6 secondary market. The ERF is purchasing real and additional abatement at a reasonable cost 7 (Climate Change Authority, 2017). The Regulator is administering the ERF reasonably well 8 and with a high level of compliance (Australian National Audit Office, 2016, Climate Change 9 Authority, 2017). However, ERF projects have high transaction costs and ERF contracts are concentrated with large carbon service providers (CSPs) (Climate Change Authority, 2017). The ERF is also a complex scheme, particularly in its MRV, that favours aggregation and CSPs at the expense of its smaller participants (Burke, 2016; Verschuuren, 2017). Concerns are also emerging that the ERF purchasing component may be headed for a future ACCU shortfall, because the projects assigned to ERF contracts are decoupled from the ACCUs surrendered to the Regulator (Climate Change Authority 2017). A poorly functioning secondary market compounds this concern. The secondary market is opaque, illiquid and shallow (Climate Change Authority, 2017). This results in limited price discovery and investment signals as ACCU supply and demand are largely unknown (Climate Change Authority, 2017). To resolve these issues more transparent secondary market information is required to strengthen the Australian carbon market (Climate Change Authority, 2017).

\subsection{Carbon market actors}

\subsubsection{Government}

The Government's role in the market is split between a department that focuses on policy settings and the Regulator that administers the market. The Government department develops the policy settings, legislation and ERF methods (Climate Change Authority, 2017). The department is supported by an independent expert committee, the Emissions Reduction Assurance Committee, that advises on whether the ERF methods meet environmental integrity standards (Climate Change Authority, 2017). The Regulator is responsible for administering the ERF. This includes registering ERF projects, verifying ERF projects claims and issuing ACCUs, ERF purchasing, and any compliance and enforcement actions 
1 also has responsibility for managing and administering the Australian National Registry of

2 Emissions Units (ANREU) accounting system that underpins the Australian carbon market.

3

4

5

6

7

8

9

10

11

\subsubsection{Project proponents}

Project proponents have the responsibility and legal right to carry out ERF projects (Climate Change Authority, 2017). These project proponents generate ACCUs, can enter into ERF contracts with the Regulator, and trade ACCUs on the ANREU.

\subsubsection{Carbon service providers}

Carbon service providers (CSPs) perform intermediary and advisory functions within the market. These roles include developing projects, advising project proponents through project registration and MRV processes, and aggregating carbon projects (Climate Change Authority, 2017; Verschuuren, 2017). These CSPs also perform market making functions such as promoting the ERF, collecting market intelligence and brokering transactions (Climate Change Authority, 2017).

\section{Australian Carbon Market Blockchain Design (ACMBD)}

\subsection{Decentralisation}

\subsubsection{Trusted authority}

In the Australian carbon market, the Regulator is the trusted authority responsible for administering the carbon market. These centralised functions include running the ANREU and ERF administration. The centralised administration of the ANREU presents a foundation to decentralise the Regulator's functions.

The ANREU is the accounting system that underpins the Australian carbon market through recording ACCU issuance, holdings and retirement. The Regulator has legislative responsibilities to manage and administer the ANREU. These responsibilities include keeping an electronic registry, administering participant eligibility, publishing selected registry information, and making corrections (Commonwealth of Australia, 2016). These responsibilities have been realised in a centralised ANREU where the Regulator manages its 
1 information, transactions, ACCU issuance and registry updating. The following section

2 considers how and whether these functions could be decentralised.

3

\section{$4 \quad 5.1 .2$ How to decentralise}

5

\section{$6 \quad$ 5.1.2.1 ANREU information}

7 Information management on the ANREU is centralised to the Regulator. The Regulator grants ANREU accountholders the ability to read their transaction history and information about credits held such as credit type, ERF project ID, Vintage, serial range and quantity (Clean Energy Regulator, 2018g). The Regulator publishes select additional information about accountholders, alterations to the registry, voluntary unit cancellation and Kyoto reporting requirements (Clean Energy Regulator, 2018e).

The literature proposes different models of managing ledger information. The Technolibertarian model proposes a completely public, open and decentralised ledger where every participant can access the complete ledger record (Al Kawasmi et al., 2015). In contrast, the Industry 4.0 and Networked Carbon models embrace a permissioned system, where actors can only view particular parts of the ledger (Macinante, 2017, Fu et al., 2018).

It is recommended that ledger information be decentralised by following the

21 Technolibertarian model. The Technolibertarian model provides each member with a copy of the ledger that can be read without restriction. This is preferred because it introduces full transparency to the opaque Australian carbon market. There are no legislative barriers to enabling this transparency. The transparency would combat the market's existing asymmetric information and illiquidity issues while increasing market scrutiny. Whereas adopting the Industry 4.0 model and Networked Carbon model would entrench the opaque secondary carbon market.

\subsubsection{ANREU transactions}

In the Australian carbon market there are three sources of ACCU transactions. These are public purchases through the ERF, private purchasing through the secondary market and voluntary cancellations. The Regulator is involved in each transaction. Each transaction follows an ANREU credit transfer protocol (Clean Energy Regulator, 2018g). This protocol starts with the accountholder selecting units to transfer, selecting a receiving account and 
1 then submitting the transaction for approval. The Regulator then provides a PIN to the accountholder to transfer the credit and complete the transaction. The transaction is then

The literature identifies two options of how this Regulator transaction role could be decentralised. The first approach in the Technolibertarian model uses an enhanced Bitcoin transaction protocol. Each party to the transaction uses a digital signature to conduct the transaction. This is then processed through an automated facilitator function that validates the transactions (see IEEE, 2000). The Networked Carbon model proposes an alternative model using smart contracts to conduct transactions. These smart contracts would have a dual structure, reflecting scheme eligibility conditions and transaction specific conditions (Macinante, 2017).

It is recommended that the Networked Carbon model's smart contracts be used to undertake ANREU transactions and decentralise the Regulator's role. The Networked Carbon model is preferred because it offers more functionality than the Technolibertarian model. The Technolibertarian model employs primitive functionality that is more simply and effective employed in the Networked Carbon model. The Networked Carbon model's smart contracts are proposed to be adapted to the ANREU by omitting the eligibility conditions. These eligibility conditions are omitted because the ANREU already deals with eligibility through a separate process. To conduct an ANREU transaction there would be a simple smart contract protocol with transaction specific requirements. These transaction specific requirements would include that the transferrer holds the ACCUs and that both the transferrer and transferee digitally sign the transaction. This would enable ACCU transactions to proceed without Regulator involvement.

\subsubsection{ACCU issuance}

The issuance of the ACCUs is centralised in the Regulator. This is the culmination of the Regulator's administration of ERF projects. Under the ERF process, project proponents register with the Regulator, conduct their project according to an ERF method, before reporting under the ERF method to the Regulator. The Regulator then undertakes a risk-based verification of the report before issuing an ACCU certificate of entitlement and issuing the ACCUs on the ANREU. 
1 The literature identifies two relevant models to decentralise a centralised carbon credit issuance process. The Industry 4.0 and Technolibertarian models propose an automated process using data from smart devices to generate carbon credits. In the Technolibertarian model this is issued autonomously without an authority, whereas in Industry 4.0 this is still managed by an authority. The second model is the Voluntary Offset model. Under this model carbon credits are issued within the DAO smart contracts. The verification process is administered manually by another University to provide assurance.

It is recommended to generate ACCUs using smart contracts. This is permissible under the Regulator's computerised decision-making powers already in the CFI Act (Commonwealth of Australia, 2017a). Using smart contracts would effectively program the existing project ACCU issuance process and remove the Regulator. The Australian carbon market possesses important building blocks that are transferable to smart contracts. These building blocks are legislated eligibility conditions and mature standardised methods with prescribed rules that projects must follow. To enter into the smart contract the project proponent would need to prove and maintain their eligibility through existing processes. The proponents would then enter into a 'Project Smart Contract' programmed with their selected method's conditions. The project would then report its data into its Project Smart Contract. As the data is fed into the Project Smart Contract, and the method's conditions are met, ACCUs would be automatically issued onto the ANREU. Projects could report their data into the Project Smart Contract using smart devices or manually. There are already ERF precedents of using smart devices to automate data collection (Verschuuren, 2017). Both automated and manual data reporting would be subject to the existing auditing arrangements. To incorporate the audits, the audit schedule would be programmed into the Project Smart Contract. At the programmed intervals auditors would verify the project before further ACCUs could be issued. In between these audits the projects would report and generate ACCUs.

\subsubsection{Updating the ANREU}

In the Australian carbon market it is the responsibility of the Regulator to update the ANREU. The Regulator is required to keeping an electronic registry and records its entries (Commonwealth of Australia, 2016, Clean Energy Regulator, 2016).

There are two options for decentralising ledger updates. The Technolibertarian model considers the adoption of Bitcoin's proof-of-work protocol. This includes creating network 
1 nodes, known as miners, which collect transactions, solve cryptographic puzzles and earn rewards. The Voluntary Offset model adopts Ethereum's similar proof-of-work protocol. In contrast, the Industry 4.0 adopts the proof-of-authority consensus protocol under the Multichain blockchain (see Greenspan, 2015). This proof-of-authority protocol is additional to the design options outlined in $\mathrm{Xu}$ et al. (2017). In the proof-of-authority protocol nominated 'authorities' take turns updating the registry (Tasca and Tessone, 2017).

It is recommended that the Australian carbon market adopt a proof of authority protocol. The Regulator would be nominated as the sole authority to update the ANREU. This would enable the Regulator to meet its legislated responsibilities under a blockchain design. It would also retain future flexibility as additional authorities can be added over time. The alternative proof-of-work protocol is problematic. Under the proof-of-work protocol the mining nodes would effectively be responsible for the recording of transactions and the keeping of the registry. This would contravene the Regulator's legislative responsibilities. The proof-of-work protocol's reliance on energy use to fuel its computation also creates a carbon emission externality that could undermine the integrity of the market (Dong et al., 2018).

\subsubsection{ERF contracts}

ERF contracts represent a special case for decentralisation of the Regulator's functions. ERF contracts primarily function as off-take agreements, whereby projects are contracted to deliver ACCUs in the future. To enter into contracts, projects must meet the ERF eligibility and offset method requirements and satisfy a number of contractual conditions (Clean Energy Regulator, 2018k).

The administration of these ERF contracts could be decentralised to an overarching 'ERF Smart Contract'. The ERF Smart Contract would contain the existing ERF contractual conditions, the Project Smart Contract of 5.1.2.3 and the smart contract transaction protocol of 5.1.2.2. To enter into the contract, the ERF contractor would have to fulfil the ERF contract conditions, nominate the Project Smart Contract(s) and specify the delivery schedule. As the project earns ACCUs, via the Project Smart Contract, they would be automatically transferred to the Regulator's nominated account via the smart contract transfer protocol. In the event of deliver shortfalls that activate make-good obligations, the ERF contractor would need to transfer ACCUs to the Regulator's nominated account before 
1 further ACCUs issuance and transfers could occur under the ERF Smart Contract. As a result the ERF purchasing would become automated and decentralised, and in the process remove the Regulator's current conflict of interest of issuing and purchasing it's own ACCUs.

4

\subsubsection{Permissions}

6 The Australian carbon market already has permissions. These permissions include what can

7 be read on the registry, who can conduct transactions on the registry and update the ANREU.

8 The Regulator also has a series of additional ANREU management powers including corrections to the Registry, suspension of accounts and the ANREU, transaction deferral and account suspension (Commonwealth of Australia, 2016).

Permissions are also an important consideration for blockchain applications. The Industry 4.0 model introduces use of finely grained permissions to restrict what participants can access and do within the blockchain. The Networked Carbon model's permissions are managed by a market regulator (Jackson et al., 2017). In contrast, the Technolibertarian model is an open permission-less system.

It is recommended that the Networked Carbon model of permissions managed by the market regulator be applied to the Australian carbon market. The Regulator would continue to manage these permissions on the network. The functions without permissions (i.e. unrestricted) would be reading the ANREU and conducting transactions. The Regulator's existing permission to generate ACCUs would be decentralised into smart contracts. Permissioned functions would include the Regulator as the only actor to update the ANREU, and the Regulator would retain its legislated ANREU enforcement and compliance functions to suspend actors, enforce obligations and cancel accounts.

\subsubsection{Verifier}

28 In the Australian carbon market, the Regulator and auditors verify carbon projects. The Regulator verifies all ERF project reports before ACCU issuance. Over time this has become a more routine process with a heavy reliance on auditors (Australian National Audit Office, 2016, Verschuuren, 2017). Auditing is determined by an auditing schedule established by the Regulator (Clean Energy Regulator, 2017), and the Regulator can also request compliance audits (Clean Energy Regulator, 2018b). These auditors must be a registered greenhouse and 
energy auditor under the National Greenhouse and Energy Reporting Act 2007 (Clean

2 Energy Regulator, 2017).

3

4 There are roles for verifiers in the blockchain carbon markets models. In the Industry 4.0

5 model verifiers are considered through an auditing role. The model carves out verifier roles to

6 evaluate firms, projects and product emissions. This also includes a reporting back function

7 to the affected firms and authority. Within the Industry 4.0 verifier roles are ad hoc (Khaqqi

8 et al., 2018) or through a single standing committee (Fu et al., 2018). The Voluntary Offset

9 model engages Universities to verify the project emissions on an ad hoc basis.

It is recommended that the Australian carbon market blockchain include verifiers. This would support the Project Smart Contracts in 5.1.2.3 that incorporate the existing audit arrangements. The existing register of greenhouse and energy auditors would be designated as trusted verifiers under the blockchain. Consistent with existing arrangements these verifiers would be used on an ad hoc basis for Project Smart Contracts. The single standing committee of auditing proposed in the Industry 4.0 is not recommended because it would create an avoidable administrative bottleneck and restrict auditing competition in the market. This verifier model combined with the Project Smart Contract effectively removes the Regulator from project MRV except for compliance actions.

\subsection{Storage and computation}

\subsubsection{Storage}

24 In the Australian carbon market, item data are distributed across multiple registries. The ANREU contains accountholder information about transaction history, and holding specific information about credit type, ERF project ID, Vintage, serial range and quantity (Clean 27 Energy Regulator, 2018g). Other information related to the market is stored in the ERF project register and the Carbon Abatement Contractor (CAC) Register. The ERF project register information includes project proponent details, method used, project description and area, and ACCUs issued over time (Clean Energy Regulator, 2018f). The CAC register includes contractor and project details, ACCU volume committed and supplied to date, delivery period and contract duration (Clean Energy Regulator, 2018d). 
1 Item data and item collection are not directly addressed in the materials examined in this

2 study. Only the Networked Carbon model indirectly considers these points by suggesting that

3 the system should be flexible enough to store metadata with emission units (Jackson et al.,

4 2017) and potentially include co-benefits information with the emissions units (Dong et al.,

5 2018).

7 For the Australian carbon market, it is recommended that the data and information already 8 present with ACCUs on the ANREU be stored on chain. Storing this data on-chain, combined 9 with the permission-less ledger reading of in 5.1.2.1, would introduce more transparency to carbon market transactions and reveal important information for the secondary market. It is also recommended to store additional information captured in the Project Smart Contracts and ERF Smart Contract on-chain. This would compile the dispersed information stored on the ERF project and CAC registers into the ANREU. Projects would also be able to store limited additional information about their ACCUs such as co-benefit information with their ACCUs.

\subsubsection{Computation}

In the Australian carbon market, computation is limited to the ANREU transaction protocol identified in 5.1.2.2.

The literature outlines different computation complexities. This ranges from the relatively simple Bitcoin based protocol of the Technolibertarian model, to additional transaction restrictions in the Industry 4.0 model, to transaction based smart contracts in the Networked Carbon model, to complex smart contracts that create DAOs in the Voluntary Offset model.

It is recommended that on-chain smart contracting be used. In the decentralisation decision of 5.1.2 smart contracts were identified as a mechanism to enable the decentralisation of the ACCU issuance and transactions. To support this design the blockchain application must possess computation sophisticated enough to process these smart contracts. The Networked Carbon model computation is recommended for consistency with the arrangements in 5.1.2. Storing these contracts on-chain is recommended to promote transparency, track smart contracts within the ANREU and allow the Regulator oversight of all smart contracts. It would also promote interoperability on-chain that supports the ERF smart contracts in 
1 5.1.2.5. The off-chain alternative would create a shadow system that undermines the 2 increased transparency of the recommended blockchain.

3

\subsection{Blockchain configuration}

5

\subsubsection{New blockchain}

7 The Australian carbon market operates using the ANREU accounting system. It is not part of an existing blockchain.

There are different options to create new blockchains or leverage off existing blockchains. The Technolibertarian, and Industry 4.0, Networked Carbon models propose creating new a blockchain. Whereas the voluntary carbon offset model proposes operating on top of the Ethereum blockchain.

It is recommended to create a new blockchain for the Australian carbon market. Creating a new blockchain would enable the Regulator to continue to meet its legislative responsibilities to keep the electronic registry. In contrast, adopting the voluntary carbon market model on top of the Ethereum blockchain would introduce doubt about whether the blockchain is actually kept by the Regulator.

\subsubsection{Multiple blockchains}

22 The Regulator currently maintains the ANREU, alongside other carbon market databases. 23 These databases are the ERF offset register and CAC register. The ANREU is also indirectly 24 linked to other international registries through the United Nations' transaction log (Clean 25 Energy Regulator, 2015b). These registries represent potential opportunities for incorporating 26 multiple blockchains.

28 Multiple blockchains could be part of a carbon market application. In the Networked Carbon 29 model, a 'federated' approach of smaller blockchains connected to large scale blockchains is outlined. The other models rely on a single blockchain.

32 It is recommended that a single blockchain be used to manage the scheme. This single 33 blockchain would house the ANREU registry and cover ACCU trading. The solution avoids 34 unnecessary duplication and reconciliation of multiple ledgers of the Networked Carbon 
1 model. Creating multiple blockchains for the ERF and CAC registries is redundant as the data 2 is already incorporated through 5.2.1. Existing arrangements with the United Nations' 3 transaction log would be maintained.

4

\section{$5 \quad$ 5.3.3 Blockchain type}

6 The Australian carbon market includes legislated restrictions on who can participate in it.

7 Participants must pass fit and proper person tests administered by the Regulator to access the 8 ANREU (Clean Energy Regulator, 2018g). This effectively makes the ANREU a private 9 ledger.

Both public and private blockchains are proposed by the different market models. The Technolibertarian model proposes an open public blockchain. The Networked Carbon, Industry 4.0 and Voluntary Offset models all propose versions of the private blockchain.

It is recommended that the Australian carbon market is a private blockchain. This is consistent with the existing eligibility requirements under the legislation that make the ANREU a private ledger. Applying a public blockchain model would contravene the legislated eligibility requirements in the Australian carbon market. To implement this private blockchain the Regulator would retain its eligibility and access management role.

\subsubsection{Data structure}

In the current Australian carbon market, the specific data structure of the ledger is not 23 publicly known.

Data structure is not explicitly addressed by any of the four market models. All models adopt by default the blockchain data structure of batching transactions into blocks.

It is recommended that the default blockchain data structure be adopted. The rationales for deviating from the default structure such as scalability and avoiding forks (Xu et al., 2017; Tien Tuan Anh et al., 2018) are unlikely to be applicable for the Australian carbon market. This is because the Regulator is the sole updater, which protects against forks, and the illiquidity of the existing carbon market is unlikely to pose scalability challenges.

\subsubsection{Consensus protocol}


1 A proof-of-authority consensus protocol is recommended as per the decentralisation 2 discussion in 5.1.2.4.

3

\section{$4 \quad$ 5.3.6 Protocol configuration}

5 In the current Australian carbon market, transactions are processed as they are conducted

6 without delay (Clean Energy Regulator, 2018j).

8 Only the Technolibertarian model considers consensus protocol configuration. The model proposes reducing the generation rate of Blocks from 10 minutes to 2.5 minutes to improve

10

11

performance and scalability.

The paper recommends that the blockchain updating frequency and size be configured to demand within the Australian carbon market. As the Regulator is recommended as the sole ANREU updater, it will be able to tailor the processing and size of blocks to meet transaction volume. The volume of transactions is likely to be low, given the concerns about liquidity on the secondary market (Climate Change Authority, 2017). The rationale for security changes to the protocol on the basis of protecting the consensus from other miners (Xu et al., 2017), is not binding as the Regulator is proposed as the sole updater.

\subsection{Other design decisions}

\subsubsection{Anonymity}

In the Australian carbon market the identities of ANREU accountholders are known and published (Clean Energy Regulator, 2018i). This is a legislated requirement (Commonwealth of Australia, 2016). However only particular transactions of these accountholders are published such as the voluntary cancellation and Kyoto credit holdings (Clean Energy Regulator, 2018i). ACCUs holdings and most transactions are not published.

Different models of anonymity are articulated. The Technolibertarian model is completely anonymous. This full anonymity only applies to identity; transactions are still visible to the network. Whereas the Networked Carbon, Industry 4.0 and Voluntary Offset models have known identities but restrictions over reading transactions. 
1 For the Australian carbon market it is recommended that the identities of participants within

2 the market continue to be known. Adopting the Technolibertarian model and anonymising the

3 identities of ANREU accountholders would conflict with the legislated requirements.

4 Combined with the permission-less reading of the ledger in 5.1.2.1 this would create

5 complete transparency in the market.

\subsubsection{Incentive}

8 In the Australian carbon market, the Regulator manages the ANREU as a public good.

In the four market models, incentives are present in the Networked Carbon and Voluntary Offset models. These models rely on proof-of-work consensus protocols that use financial incentives of token generation and transaction fees to align miner self-interest with responsible behaviour.

It is recommended that the Australian carbon market blockchain continue to operate as a public good. With the Regulator as the sole updater of the ledger there is no need to introduce incentives to promote responsible mining behaviour.

\subsubsection{Deployment}

20 The ANREU is currently deployed through a portal on the Regulator's website (Clean Energy 21 Regulator, 2018c). The ANREU was built by a third party following a tendering process 22 (Clean Energy Regulator, 2015a).

All four market models consider blockchain deployment as leveraging off existing blockchains. The Industry 4.0 model proposes using multichain blockchain, and the Voluntary Offset model prefers Ethereum. The Technolibertarian model is based on Bitcoin, 27 but it is unclear whether it will be deployed on Bitcoin infrastructure. The Networked Carbon model does not propose a specific model but notes blockchain IT functionality should be able to incorporate future changes (Dong et al., 2018).

It is recommended that the Regulator go out to tender to select a blockchain deployment solution. This is the same process that occurred with the ANREU's development. It is recommended that as part of the deployment considerations that the capacity to incorporate 
1 future blockchain functionality is considered. Ultimately the deployment decision would be a matter for Government consideration.

3

Table 2 here - Recommended Australian carbon market blockchain design

5

6

\section{Discussion}

The recommended Australian carbon market blockchain design (ACMBD) in Table 2, is a partially decentralised, private, smart contract enabled Blockchain. The ACMBD offers the Australian carbon market increased transparency, reduced participation costs and disintermediation. Implementing this ACMBD would likely create a positive effect on the efficiency, equity, and effectiveness of the Australian carbon market. These criteria are established principles for evaluating the Australian carbon market (Commonwealth of Australia, 2015) and climate change policy (Stern et al., 2006).

\subsection{Efficiency}

The ACMBD introduces transparency into the Australian carbon market that can improve economic efficiency. The existing secondary market is illiquid, shallow and inefficient (Climate Change Authority, 2017). This market is inhibited by poor information on ACCU holdings and trading, that distorts investment and price signals (Climate Change Authority, 2017). Increased transparency has been recommended to improve the market's functioning (Climate Change Authority, 2017). The ACMBD would provide this transparency through its combination of unrestricted ANREU data and enhanced on-chain ACCU information. This combination would result in more information on the supply of already committed ACCUs in ERF contracts and uncommitted ACCUs. This would help facilitate trading and provide a clearer investment signal for ERF project development. The increased transparency would also help address asymmetric information at the transaction level. Parties currently have little information about a counterparty's reputation and ACCU quality. Through the ACMBD counterparty reputations could be established by reviewing the ANREU data and their transactions. Similarly, ACCUs could be traced back to their issuance. This traceability would combine with the enhanced ACCU information to provide prospective purchasers with better information to determine the quality of ACCUs. The ACMBD's increased 
1 transparency will need to be balanced against potential concerns from market participants on the availability of data that may be considered commercially sensitive.

3

4

The ACMBD could improve the efficiency of the Australian carbon market through reducing transaction costs. The largest potential is in automation of the manual MRV of ERF projects. Currently the MRV process is costly, time consuming and dominated by CSPs (Climate Change Authority, 2017). Through the creation of the Project Smart Contracts, the MRV would become more automated in data collection and reporting. This automation would reduce the need for CSPs to collect data and save costs for project proponents. There is already a successful precedent of project proponents saving these MRV costs through automated data collection using smart devices under the piggeries methane method (Verschuuren, 2017). The Project Smart Contracts also offer automated reporting and generation of ACCUs. This automated reporting means that project proponents would no longer need CSPs for its bureaucratic reporting process. This would further reduce the need for CSPs and save project costs. The Project Smart Contracts would also cut the ACCU issuance timelines. Currently it takes the Regulator on average six weeks to assess a report for ACCUs (Clean Energy Regulator, 2018h). Through the Project Smart Contract this assessment and issuance would be done instantly. The reduction in project costs and time is likely to encourage more frequent project reporting. More frequent reporting will improve project cash flow and promote market liquidity as ACCUs become available more often. These smart contract benefits will need to be balanced against the initial set up costs of the Project Smart Contracts. There will be costs in developing the new contracts, transitioning existing arrangements and project proponent education.

\subsection{Equity}

The ACMBD could promote equity through improved transparency. The existing Australian carbon market is inequitable as it is overwhelmingly concentrated in the hand of a few CSPs service providers. In 2017, 74 per cent of ERF contracted projects were attributable to CSPs, with two CSPs controlling 55 per cent of all ERF contracts (Climate Change Authority, 2017). The ACMBD could make this more equitable by eroding the CSPs' information advantage. Under the existing Australian carbon market, information is hoarded by CSPs. This enables the CSPs to maximise their chances to secure ERF contract funding. As CSPs have better information they can better calibrate ERF reverse auction bids to maximise their 
1 price and likelihood of securing funding. The ACMBD's transparency would increase the 2 availability of this hoarded market information. This would level the playing field for the 3 ERF reverse auction by giving all ERF projects a shared information base. This is likely to encourage a more competitive reverse auction that may also reduce the costs of Government ACCU purchases that in turn may lead to more ACCUs being contracted for a given ERF funding allocation.

ACMBD's reduced transaction costs also challenge the rationale for the existing CSP intermediation. CSPs currently perform intermediary roles in brokering transactions, MRV processes and aggregating projects. CSPs are able to broker transactions through their market informational advantages. With a transparent ANREU this advantage is eroded. Accountholders ean view the entire ANREU and identify uncommitted ACCUs for purchase without a CSP broker. The reduced MRV transaction costs, outlined in 6.1, are another potential source of disintermediation as CSPs are relegated by Project Smart Contracts. The CSP aggregation role is also challenged from falling transaction costs. ERF aggregation relies on bundling up smaller projects to create economies of scale in the face of high transaction costs. With lower transaction costs under the ACMBD, there is a potential disaggregation as smaller projects become more viable and new projects emerge that were previously unviable under the higher transaction costs. This disaggregation may also help allay concerns of unscrupulous CSPs and CSP aggregators unfairly burdening unsuspecting landholders with project risk (Climate Change Authority, 2017, Verschuuren, 2017). As CSPs are relegated there may be a trade-off with other useful CSP functions. These useful CSPs functions have included promoting the Australian carbon market, developing projects and providing advice to projects proponents. If there is significant CSP disintermediation then these functions are likely to not occur to the same extent.

\subsection{Effectiveness}

The ACMBD can lead to more effective regulation to support the delivery of genuine emission reductions. Currently to ensure environmental effectiveness the Regulator is extensively involved in the ERF project process. The Project Smart Contract creates an opportunity for the Regulator to engage in smarter regulation. Under the Project Smart 33 Contracts, ACCUs are generated according to meeting the method's rules. This effectively programs the Regulator's role in checking that projects meet their ERF method requirements. 
1 It does not however, sacrifice the quality of project verification as the ACMBD retains the audit assurance function that the Regulator relies upon (Verschuuren, 2017). The Project Smart Contracts establish objective rules that would prevent projects from earning ACCUs unless the rules are met. This would address existing concerns about the Regulator's level of transparency and consistency in decision-making by introducing transparent objective standards (Climate Change Authority, 2017). The Project Smart Contracts would also support smarter regulation through real-time regulation. The ACMBD would enable the Regulator to have a new level of oversight through the transparent Project Smart Contracts. The Regulator could see how the project is tracking in real time instead of the current periodic reporting. This would allow the Regulator to address poor compliance and intervene earlier where required. Ultimately the effectiveness of this regulation rests on the development of robust Project Smart Contracts that reflect the ERF methods. The Regulator would need to work with the Department and its Emissions Reduction Assurance committee to develop these robust smart contracts.

The ACMBD, through increased transparency is likely to support the delivery of genuine emissions reductions within the Australian carbon market. Currently the decoupling of ERF projects from the ERF contracts is creating a situation where there is a risk of ERF delivery shortfalls (Climate Change Authority, 2017). This undermines the effectiveness of the scheme. Under the ACMBD the Regulator would have a new level of oversight of the delivery of ACCUs under the ERF Smart Contracts. This would be supported by the ACMBD's transparency that leads to a better understanding of market ACCU supply. Together this would allow the Regulator to better assess its delivery risk. With a better risk assessment the Regulator could undertake more proactive mitigation strategies including contracting more projects or signalling to the market to develop more projects. Ultimately this would help ensure that emissions reductions are delivered under the ERF.

\section{Conclusion and policy implications}

This paper created a blockchain design for the Australian carbon market (the ACMBD). The paper systematically developed the ACMBD by assessing four existing blockchain models derived from previous literature, for their applicability in the context of the Australian carbon market. The ACMBD is configured to the existing legislation and institutions of the 
1 Australian carbon market. The paper proposes that implementing the ACMBD could improve 2 the Australian carbon market's performance. The ACMBD could improve economic 3 efficiency by creating a more transparent secondary market and reducing transaction costs.

4 The Australian carbon market could become more equitable through increased transparency 5 and disintermediation. Finally, the ACMBD could through smarter regulation and more 6 informed ERF management improve the environmental effectiveness of the scheme.

7

8 This paper's findings should be considered in the context of its limitations. As a desktop 9 review, using document analysis, the paper is dependent on the availability of blockchain and carbon market research. As blockchain is a new phenomenon, there was only limited available literature to draw upon. The paper is also a conceptual analysis of applying the blockchain to the Australian carbon market. Future research should move beyond conceptual analyses by engaging with blockchain and Australian carbon markets experts to test the recommended design. As part of this testing the practical implementation challenges and costs of the recommended ACMBD should be explored. The paper is also a point in time analysis of the Australian carbon market and is constrained by its existing market rules, legislation and institutions. Future research should consider how legislation and institutions could evolve to support blockchain. This is especially pertinent as the Australian carbon market is likely on the verge of another incarnation with ERF funds nearly exhausted. Finally, this research focused on the Australian carbon market; future research should consider blockchain applications for other existing carbon markets.

This paper has made an important contribution by filling a clear research gap in how blockchain could be applied to an existing carbon market. The paper provides a new method for climate change policymakers to consider blockchain applications for carbon markets. It also makes a timely contribution to Australian climate change policy by introducing a model that could potentially improve its efficiency, equity and effectiveness. Ultimately it is hoped that this research could make a contribution as a bridge between existing carbon markets, new technology, and the future carbon market arrangements under the Paris Agreement. 
AL KAWASMI, E., ARNAUTOVIC, E. \& SVETINOVIC, D. 2015. Bitcoin-Based Decentralized Carbon Emissions Trading Infrastructure Model. Systems Engineering, $18,115-130$.

ALKETBI, A., NASIR, Q. \& TALIB, M. A. Blockchain for government services - Use cases, security benefits and challenges. 2018 15th Learning and Technology Conference (L\&T), 25-26 Feb. 2018 2018. 112-119.

ASTE, T., TASCA, P. \& DI MATTEO, T. 2017. Blockchain Technologies: The Foreseeable Impact on Society and Industry. Computer, 50, 18-28.

AUSTRALIAN NATIONAL AUDIT OFFICE. 2016. Abatement Crediting and Purchasing under the Emissions Reduction Fund [Online]. Available: https://www.anao.gov.au/sites/g/files/net5496/f/ANAO_Report_14_2016-17.pdf [Accessed 8 September 2018].

BOURI, E., MOLNÁR, P., AZZI, G., ROUBAUD, D., \& HAGFORS, L. I. (2017). On the hedge and safe haven properties of Bitcoin: Is it really more than a diversifier?. Finance Research Letters, 20, 192-198.

BUMPUS, A. G. \& LIVERMAN, D. M. 2008. Accumulation by decarbonization and the governance of carbon offsets. Economic Geography, 84, 127-155.

BURGER, S. P., \& LUKE, M. (2017). Business models for distributed energy resources: A review and empirical analysis. Energy Policy, 109, 230-248.

BURKE, P. J. 2016. Undermined by adverse selection: Australia's direct action abatement subsidies. Economic Papers: A journal of applied economics and policy, 35, 216-229.

BUTERIN, V. 2013. Ethereum [Online]. Available: https://github.com/ethereum/wiki/wiki/White-Paper [Accessed 19 February 2018].

CATALINI, C. \& GANS, J. S. 2017. Some Simple Economics of the Blockchain. Rotman School of Management Working Paper No. 2874598: MIT Sloan Research Paper.

CLEAN ENERGY REGULATOR. 2015a. History of the ANREU [Online]. Available: http://www.cleanenergyregulator.gov.au/OSR/ANREU/History-of-ANREU [Accessed 22 October 2018].

CLEAN ENERGY REGULATOR. 2015b. Supporting the Kyoto Protocol [Online]. Available: http://www.cleanenergyregulator.gov.au/OSR/ANREU/Supporting-theKyoto-Protocol [Accessed 7 September 2018].

CLEAN ENERGY REGULATOR. 2016. Carbon Farming Initiative [Online]. Available: http://www.cleanenergyregulator.gov.au/Infohub/CFI/Carbon-Farming-Initiative [Accessed 3 October 2018]. 
1 CLEAN ENERGY REGULATOR. 2017. Auditing [Online]. Available: http://www.cleanenergyregulator.gov.au/ERF/Want-to-participate-in-the-EmissionsReduction-Fund/Step-3-Reporting-and-auditing/Audit-Requirements - Audit-frequency [Accessed 15 October 2018].

CLEAN ENERGY REGULATOR. 2018a. Auction June 2018 [Online]. Available: http://www.cleanenergyregulator.gov.au/ERF/Auctions-results/june-2018 [Accessed 8 September 2018].

CLEAN ENERGY REGULATOR. 2018b. Audit requirements for programs administered by the Clean Energy Regulator [Online]. Available: http://www.cleanenergyregulator.gov.au/Infohub/Audits/Pages/Forms and resources/Audit determination handbook/Audit-requirements-for-programmesadministered-by-the-Clean-Energy-Regulator.aspx - 143-Emissions-Reduction-Fundaudits [Accessed 22 October 2018].

CLEAN ENERGY REGULATOR. 2018c. Australian National Registry of Emissions Units [Online]. Available: https://nationalregistry.cleanenergyregulator.gov.au/ [Accessed 22 October 2018].

CLEAN ENERGY REGULATOR. 2018d. Carbon Abatement Contract Register [Online]. Available: http://www.cleanenergyregulator.gov.au/ERF/project-and-contractsregisters/carbon-abatement-contract-register [Accessed 22 October 2018].

CLEANENERGY REGULATOR. 2018e. Data and Information: Reports to support the Kyoto Protocol [Online]. Available: http://www.cleanenergyregulator.gov.au/OSR/ANREU/Data-and-information [Accessed 19 October 2018].

CLEAN ENERGY REGULATOR. 2018f. Emissions Reduction Fund Project Register [Online]. Available: http://www.cleanenergyregulator.gov.au/ERF/project-andcontracts-registers/project-register [Accessed 27 October 2018].

CLEAN ENERGY REGULATOR. 2018g. Managing units in the Australian National Registry of Emissions Units ANREU [Online]. Available: http://www.cleanenergyregulator.gov.au/DocumentAssets/Pages/Managing-units-inthe-Australian-National-Registry-of-Emissions-Units-ANREU.aspx [Accessed 8 September 2018].

CLEAN ENERGY REGULATOR. 2018h. Processing times for project registration, variation and crediting applications [Online]. Available: http://www.cleanenergyregulator.gov.au/ERF/Want-to-participate-in-the-Emissions- 

crediting-applications [Accessed 14 October 2018].

CLEAN ENERGY REGUlATOR. 2018i. Public Reports Home [Online]. Available: https://nationalregistry.cleanenergyregulator.gov.au/report/listPublicReports [Accessed 22 October 2018].

CLEAN ENERGY REGULATOR. 2 October 2018 2018j. RE: Registry Processing Times.

CLEAN ENERGY REGULATOR. 2018k. Want to participate in the Emissions Reduction Fund? [Online]. Available: http://www.cleanenergyregulator.gov.au/ERF/Want-toparticipate-in-the-Emissions-Reduction-Fund [Accessed 8 September 2018].

CLIMATE CHANGE AUTHORITY. 2017. Review of the Emissions Reduction Fund [Online]. Online. Available: http://climatechangeauthority.gov.au/review-emissionsreduction-fund [Accessed 8 September 2018].

COMMONWEALTH OF AUSTRALIA. 2008. Carbon Pollution Reduction Scheme Green Paper [Online]. Department of Climate Change. Available: https://web.archive.org/web/20080722030824/http://www.greenhouse.gov.au/greenpap er/report/pubs/greenpaper.pdf [Accessed 8 September 2018].

COMMONWEALTH OF AUSTRALIA. 2011. Securing a Clean Energy Future: The Australian Government's Climate Change Plan [Online]. Available: https://web.archive.org/web/20110715114216/http://www.cleanenergyfuture.gov.au/wp -content/uploads/2011/07/Consolidated-Final.pdf [Accessed 8 September 2018].

COMMONWEALTH OF AUSTRALIA. 2014. Emissions Reduction Fund White Paper [Online]. Available: http://www.environment.gov.au/system/files/resources/1f98a9245946-404c-9510-d440304280f1/files/erf-white-paper.pdf [Accessed 8 September 2018].

COMMONWEALTH OF AUSTRALIA 2015. Climate Change Authority Act 2011.

COMMONWEALTH OF AUSTRALIA 2016. Australian National Registry of Emissions Units Act 2011.

COMMONWEALTH OF AUSTRALIA 2017a. Carbon Credits (Carbon Farming Initiative) Act 2011

COMMONWEALTH OF AUSTRALIA. 2017b. Review of Australia's climate change policies [Online]. Available: http://www.environment.gov.au/climate-change/reviewclimate-change-policies [Accessed 8 September 2018].

DEPARTMENT OF THE ENVIRONMENT. 2016. The safeguard mechanism - Managing excess emissions [Online]. Available: http://www.environment.gov.au/climate- 
change/government/emissions-reduction-fund/publications/factsheet-safeguardmechanism-managing-excess-emissions [Accessed 8 October 2018].

DIESTELMEIER, L. (2019). Changing power: Shifting the role of electricity consumers with blockchain technology-Policy implications for EU electricity law. Energy Policy, 128, 189-196.

DONG, X., MOK, R. C. K., TABASSUM, D., GUIGON, P., FERREIRA, E., SINHA, C. S., PRASAD, N., MADDEN, J., BAUMANN, T., LIBERSKY, J., MCCORMICK, E. \& COHEN, J. 2018. Blockchain and emerging digital technologies for enhancing post2020 climate markets (English). Washington, D.C: World Bank Group.

FU, B., SHU, Z. \& LIU, X. 2018. Blockchain Enhanced Emission Trading Framework in Fashion Apparel Manufacturing Industry. Sustainability, 10, 1105-1124.

GREEN, J., \& NEWMAN, P. (2017). Citizen utilities: The emerging power paradigm. Energy Policy, 105, 283-293.

GREENSPAN, G. 2015. MulticChain Private Blockchain - White Paper [Online]. Available: https://www.multichain.com/download/MultiChain-White-Paper.pdf [Accessed 25 September 2018].

IANSITI, M. \& LAKHANI, K. R. 2017. The Truth about Blockchain. Harvard Business Review, 95, 118-127.

INSTITUTE OF ELECTRICAL AND ELECTRONICS ENGINEERS (IEEE) 2000. IEEE Standard Specifications for Public-Key Cryptography. IEEE Std 1363-2000, 1-228. JACKSON, A., LLOYD, A., MACINANTE, J. \& HÜWENER, M. 2017. Networked Carbon Markets: Permissionless Innovation with Distributed Ledgers? [Online]. SSRN. Available: https://ssrn.com/abstract=2997099 [Accessed 21 March 2018].

KHAQQI, K. N., SIKORSKI, J. J., HADINOTO, K. \& KRAFT, M. 2018. Incorporating seller/buyer reputation-based system in blockchain-enabled emission trading application. Applied Energy, 209, 8-19.

KRISTOFFER, F. \& DAVID, S. 2018. The Supply Chain Has No Clothes: Technology Adoption of Blockchain for Supply Chain Transparency. Logistics, 2.

KSHETRI, N. 2017. Will blockchain emerge as a tool to break the poverty chain in the Global South? Third World Quarterly, 38, 1710-1732.

LEONHARD, R. 2017a. Developing the Crypto Carbon Credit on Ethereum's Blockchain [Online]. SSRN. Available: https://papers.ssrn.com/sol3/papers.cfm?abstract_id=3000472 [Accessed 21 March 2018]. 
LEONHARD, R. 2017b. Forget Paris: Building a Carbon Market in the U.S. Using Blockchain-Based Smart Contracts [Online]. SSRN. Available: https://papers.ssrn.com/sol3/papers.cfm?abstract_id=3082450 [Accessed 21 March 2018].

MACINANTE, J.D. 2016. Networking Carbon Markets: Key Elements of the Process [Online]. World Bank. Available: https://openknowledge.worldbank.org/handle/10986/25750 [Accessed 12 March 2018].

MACINANTE, J. D. 2017. A Conceptual Model for Networking of Carbon Markets on Distributed Ledger Technology Architecture. Climate and Carbon Law Review, 17, 243-260.

MACKENZIE, D. 2009. Making things the same: Gases, emission rights and the politics of carbon markets. Accounting, Organizations and Society, 34, 440-455.

NAKAMOTO, S. 2008. Bitcoin: A peer-to-peer electronic cash system [Online]. Available: https://bitcoin.org/bitcoin.pdf [Accessed 11 February 2018].

NARAYANAN, A. \& CLARK, J. 2017. Bitcoin's Academic Pedigree. Communications of the ACM, 60, 36-45.

SCHMALENSEE, R. \& STAVINS, R. N. 2017. Lessons Learned from Three Decades of Experience with Cap and Trade. Review of Environmental Economics and Policy, 11, 59-79.

SELMI, R., MENSI, W., HAMMOUDEH, S., \& BOUOIYOUR, J. 2018. Is Bitcoin a hedge, a safe haven or a diversifier for oil price movements? A comparison with gold. Energy Economics, 74, 787-801.

STERN, N., PETERS, S., BAKHSHI, V., BOWEN, A., CAMERON, C., CATOVSKY, S., CRANE, D., CRUICKSHANK, S., DIETZ, S. \& EDMONSON, N. 2006. Stern Review: The economics of climate change [Online]. HM treasury London. Available: http://webarchive.nationalarchives.gov.uk/20100407172811/http://www.hmtreasury.gov.uk/stern_review_report.htm [Accessed 27 October 2018].

SUHAS, H. V., MALLA, R. \& RAVI, S. 2015. Red black cryptography. 2015 IEEE International Advance Computing Conference (IACC), 716.

TASCA, P. \& TESSONE, C. J. 2017. Taxonomy of Blockchain Technologies. Principles of Identification and Classification [Online]. Available: https://arxiv.org/abs/1708.04872 [Accessed 6 August 2018]. 
1 TIEN TUAN ANH, D., LIU, R., OOI, B. C., WANG, J., ZHANG, M. \& CHEN, G. 2018.

2

3

4

Untangling Blockchain: A Data Processing View of Blockchain Systems. 30, 13661385.

UNITED NATIONS CLIMATE CHANGE. 2017. How Blockchain Technology Could Boost Climate Action [Online]. Available: https://unfccc.int/news/how-blockchaintechnology-could-boost-climate-action [Accessed 10 May 2018].

UNITED NATIONS CLIMATE CHANGE. 2018. UN Supports Blockchain Technology for Climate Action [Online]. Available: https://unfccc.int/news/un-supports-blockchaintechnology-for-climate-action [Accessed 12 May 2018].

VERSCHUUREN, J. 2017. Towards a Regulatory Design for Reducing Emissions from Agriculture: Lessons from Australia's Carbon Farming Initiative. Climate Law, 7, 1-51. WILLIAM E, L., LUKE J, R., LIAM D, W., COLIN F, A., \& ANTHONY R, S. (2012). An economic evaluation of the potential for distributed energy in Australia. Energy Policy, 51, 277-289.

WORLD BANK \& ECOFYS. 2018. State and Trends of Carbon Pricing 2018 [Online]. Washington, DC: World Bank. Available: https://openknowledge.worldbank.org/handle/10986/29687 [Accessed 26 October $2018]$.

XU, X., WEBER, I., STAPLES, M., ZHU, L., BOSCH, J., BASS, L., PAUTASSO, C. \& RIMBA, P. 2017. A Taxonomy of Blockchain-Based Systems for Architecture Design. IEEE.

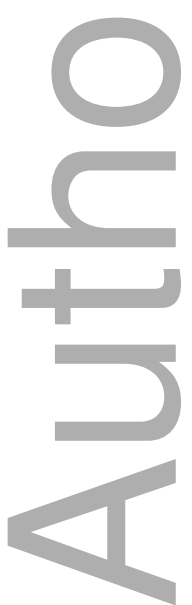


Tables

Table 1: Blockchain design decisions (Xu et al., 2017)

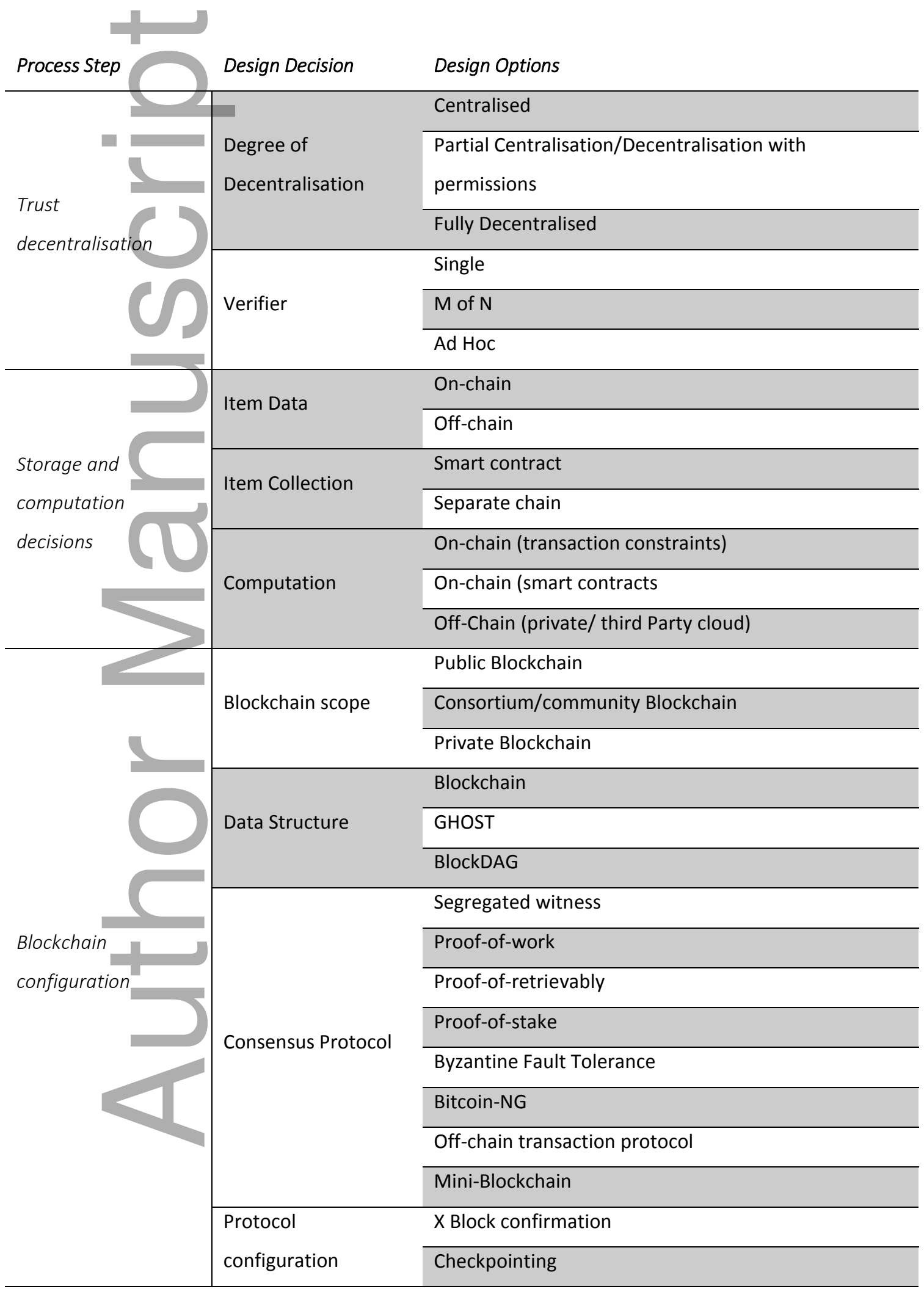

This article is protected by copyright. All rights reserved 


\begin{tabular}{|c|c|c|}
\hline \multirow{7}{*}{-1} & & Original block size and frequency \\
\hline & & Increase block size / decrease mining time \\
\hline & \multirow{5}{*}{ New Blockchain } & Merged mining \\
\hline & & Hook popular Blockchain at transaction level \\
\hline & & Proof-of-burn \\
\hline & & Side-chains \\
\hline & & Multiple private Blockchains \\
\hline \multirow{7}{*}{ decisions } & \multirow{3}{*}{ Anonymity } & Anonymity \\
\hline & & Pseudonymity \\
\hline & & Known identity \\
\hline & \multirow{2}{*}{ Incentive } & Coins \\
\hline & & Transaction fees \\
\hline & \multirow{2}{*}{ Deployment } & Cloud infrastructure \\
\hline & & Use an existing Blockchain \\
\hline
\end{tabular}


Table 2 - Recommended Australian carbon market blockchain design (ACMBD)

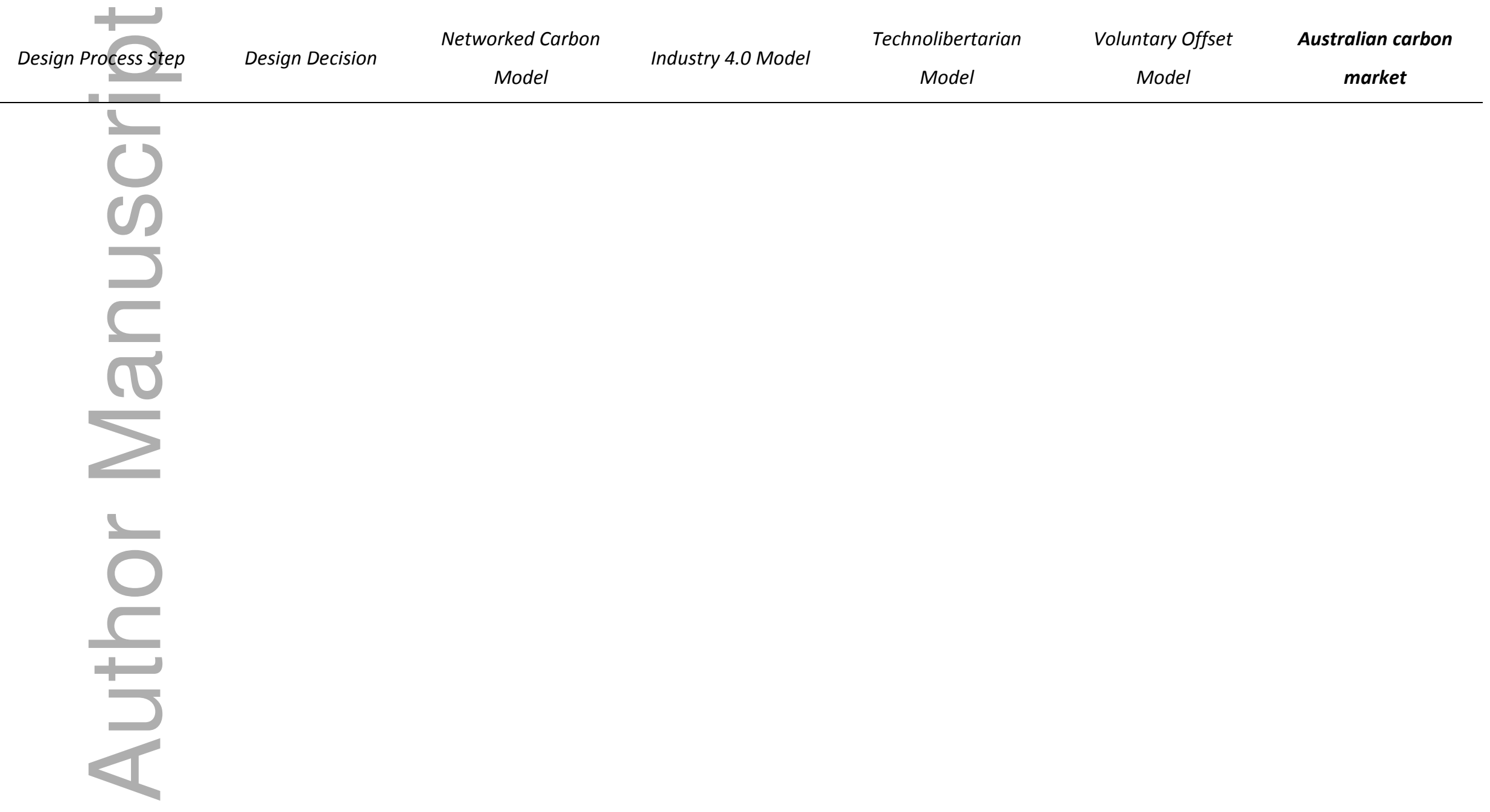

This article is protected by copyright. All rights reserved 


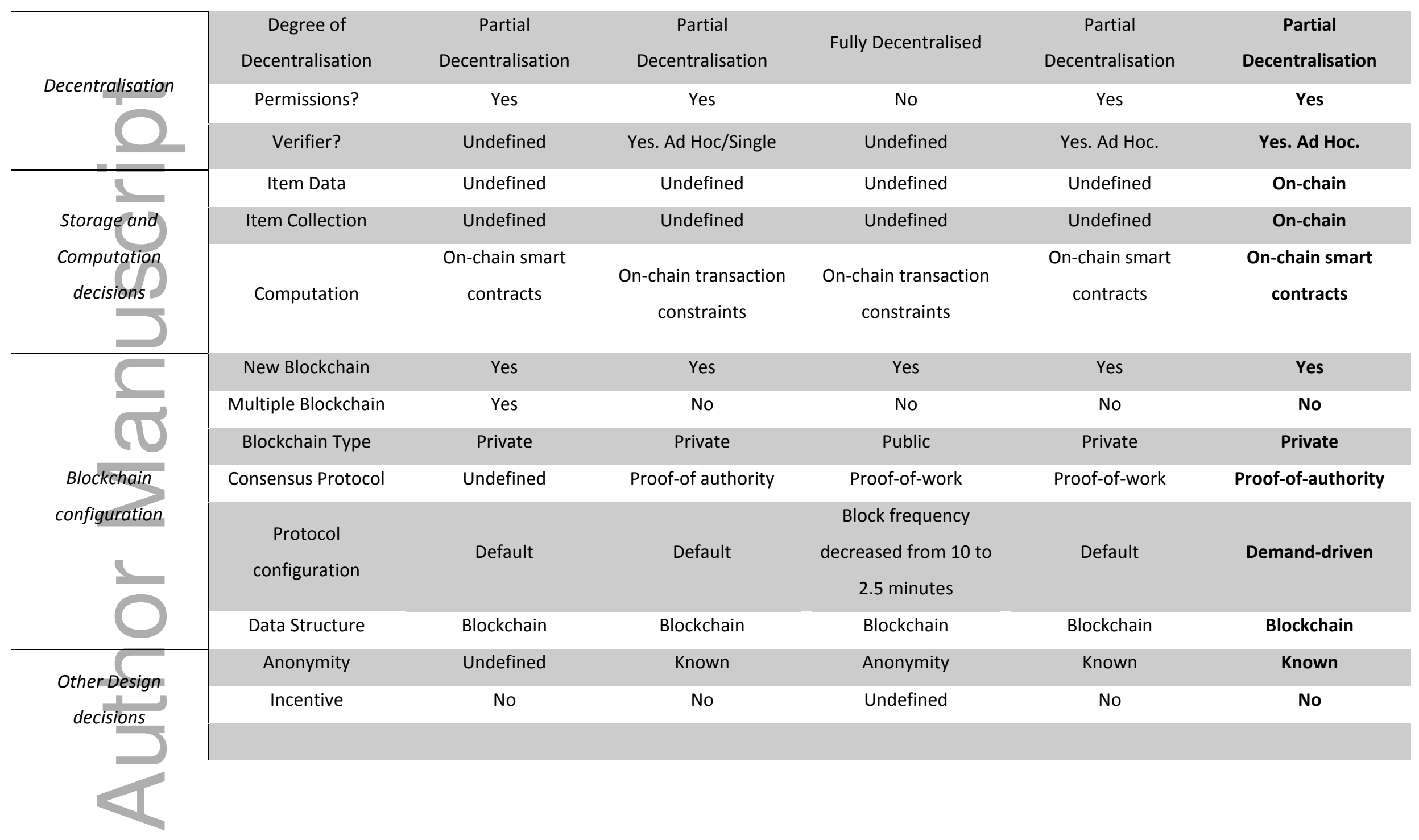

This article is protected by copyright. All rights reserved 


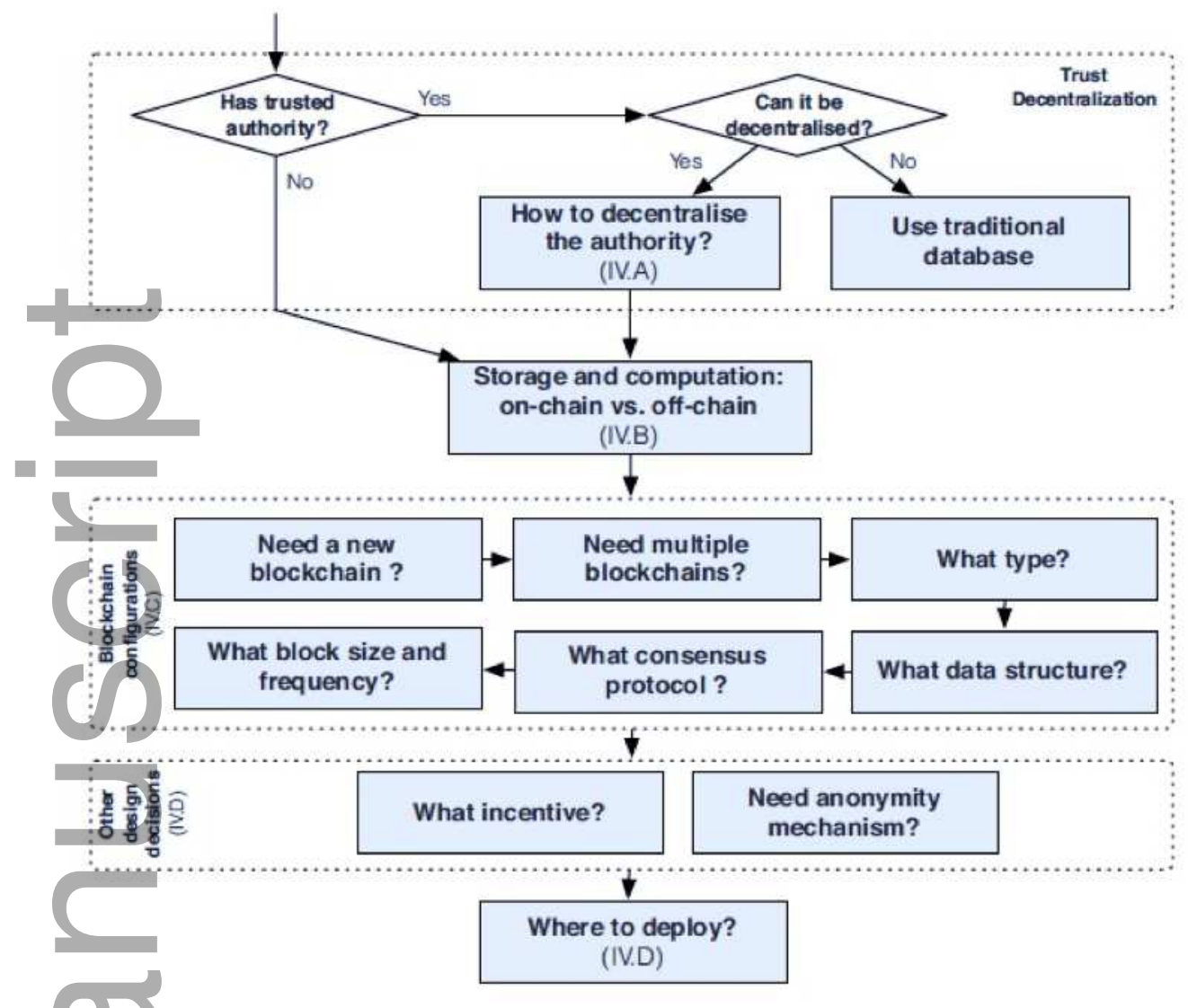

ecpa_12266_f1.png 


\section{University Library}

\section{- M M I N E R VA A gateway to Melbourne's research publications}

Minerva Access is the Institutional Repository of The University of Melbourne

Author/s:

Hartmann, S;Thomas, S

Title:

Applying Blockchain to the Australian Carbon Market

Date:

2019-08-14

Citation:

Hartmann, S. \& Thomas, S. (2019). Applying Blockchain to the Australian Carbon Market.

ECONOMIC PAPERS, 39 (2), pp.133-151. https://doi.org/10.1111/1759-3441.12266.

Persistent Link:

http://hdl.handle.net/11343/286907 